\title{
A comparison of two
} formulations of intradermal

\section{capsaicin as models of neuropathic pain in healthy volunteers}

\section{Correspondence}

Professor Paul Rolan, The University of Adelaide, 5th Level, Medical School North Adelaide, SA 5005, Australia.

Tel.: +61 883034102

Fax: +61 882240685

E-mail:paul.rolan@adelaide.edu.au

Keywords

capsaicin, cyclodextrin, volunteers

\section{Received}

25 May 2008

Accepted

24 June 2009

\section{Helena Gustafsson, Johanna Åkesson, Chai Li Lau, ${ }^{1}$ Desmond Williams, ${ }^{1}$ Lisa Miller, ${ }^{2}$ Sharon Yap ${ }^{3} \&$ Paul Rolan ${ }^{4}$}

Pharmacy School, University of Gothenburg, Gothenburg, Sweden, ${ }^{1}$ School of Pharmacy and Medical

Sciences and the Sansom Institute for Health Research, University of South Australia, ${ }^{2}$ Data

Management \& Analysis Centre and ${ }^{4}$ Discipline of Pharmacology, University of Adelaide and

${ }^{3}$ Pharmacy Department, Royal Adelaide Hospital, Adelaide, Australia

\section{WHAT IS ALREADY KNOWN ABOUT}

\section{THIS SUBJECT}

- Intradermal capsaicin has some utility as a model of neuropathic pain

- Dose-response relationships have not been well documented

- Two formulations have previously been used but their performance has not been compared

\section{WHAT THIS STUDY ADDS}

-Dose-response relationships for both formulations

- The formulations are not equivalent at al doses

- The hydroxypropyl- $\beta$-cyclodextrin formulation is preferable

\section{AIMS}

To compare the dose-response relationships of two formulations [Tween- or hydroxypropyl- $\beta$-cyclodextrin (HP- $\beta$-CD)-based] of intradermal capsaicin in healthy volunteers and to assess the effect of potential covariates of response. One, 10,30 and $100 \mu \mathrm{g}$ in $10 \mathrm{ml}$ were compared for the outcomes of flare, spontaneous pain, mechanical allodynia and hyperalgesia in eight healthy men and eight healthy women.

\section{RESULTS}

The formulations produced comparable responses at doses 1, 10 and $30 \mu \mathrm{g}$, but in all parameters the response was less at $100 \mu \mathrm{g}$ with the Tween formulation. Mean area for hyperalgesia was $9 \mathrm{~cm}^{2}[95 \%$ confidence interval $(\mathrm{CI}) 5,13$ ] higher with the HP- $\beta-C D$ formulation. Flare area was $5 \mathrm{~cm}^{2}(95 \% \mathrm{Cl} 8,13)$ greater with the HP- $\beta-C D$ formulation. There was a significant difference between pain responses from the injection site on the upper forearm compared with the lower forearm on all four pain assessments. In contrast, significant differences were seen in pain response between nondominant and dominant arm for flare, allodynia and hyperalgesia but not for spontaneous pain. A significant difference in sex was seen only for hyperalgesia. The nominal $100-\mu \mathrm{g}$ dose of the Tween formulation contained only $39 \%$ of label strength in the aqueous phase, which may explain the lower pharmacodynamic response.

\section{CONCLUSION}

The formulations are comparable over the dose range 1-30 $\mu \mathrm{g}$. The significantly lower pain response at the $100 \mu \mathrm{g}$ dose in the Tween compared with the HP- $\beta-C D$ formulation is likely to be due to limitations in solubility at the $100 \mu \mathrm{g}$ level. Given the greater ease of formulation and the superior dose-response relationship, the HP- $\beta-C D$ formulation is preferable for use in the model in future studies. 


\section{Introduction}

The use of the clinical pain state has evident limitations in evaluating analgesic drug activity. Pain states are often multifactorial with treatment regimens involving multiple medications. Evaluation of potential new analgesic agents may be helped by the use of pain models in early clinical development [1]. Volunteer pain models permit a more well-controlled study design and are useful in the study of specific pain mechanisms.

Neuropathic pain is a type of chronic pain caused by damage to or malfunctioning of the peripheral or central nervous system [2]. Intradermal capsaicin has been used as a human pain model as it replicates some of the key features of neuropathic pain:

1 Spontaneous pain, a short-lived (10-30 min) burning/ aching sensation experienced at the site of administration.

2 Allodynia, pain that is evoked by previously nonpainful stimuli. The allodynia is usually short-lived (20 $\mathrm{min}$ ) and appears as both primary and secondary allodynia.

3 Hyperalgesia, increased pain evoked by a previously mildly painful stimulus. This lasts between 6 and $24 \mathrm{~h}$ and occurs at the site of administration (primary hyperalgesia) and in the surrounding skin area (secondary hyperalgesia) [3].

4 Additionally, an axon reflex flare appears soon after injection.

Capsaicin, the active component of hot chilli pepper, is insoluble in water, causing a problem for intradermal use. A capsaicin formulation [3], which is a solution in lower concentrations and a colloidal suspension in higher concentrations, has been used extensively $[1,4,5]$ but is associated with several disadvantages. A colloidal suspension may reduce the effective local concentration, resulting in unequal given dosages, or act as a depot. The formulation is also difficult to prepare and must be freshly prepared.

Cyclodextrins are potentially useful agents for increasing the aqueous solubility of lipophilic compounds like capsaicin. They have hydrophobic inner cavities and lipophobic outer surfaces, capable of interacting with a large variety of guest molecules to form noncovalent inclusion complexes [6]. In addition to increased solubility, cyclodextrins can also improve the stability of substances against dehydration, hydrolysis, oxidation and photodecomposition, thus increasing the shelf life of the substances [7].

A study has been published in which the doseresponse relationship of capsaicin in a hydroxypropyl- $\beta$ cyclodextrin (HP- $\beta-C D$ ) formulation used in volunteers was investigated [1]. However, to our knowledge no previous study has compared the performance of such a formulation with that of the conventional formulation on which most of the clinical validation is based. In order to have confidence in the utility of a HP- $\beta-C D$ formulation of capsaicin, a clinical validation study comparing the two formulations was required.

The objectives were to compare the dose-response and dose-duration curves for spontaneous pain, flare, allodynia and hyperalgesia of two formulations of intradermal capsaicin in healthy volunteers. We also planned to examine the effect of the potential covariates sex, site of injection (upper vs. lower forearm) and dominant vs. nondominant arm.

\section{Methods}

\section{Trial design and subjects}

Sixteen healthy Caucasian volunteers, eight male and eight female, aged 19-58 years (mean 25.7), participated in this randomized, blinded, cross-over trial, after giving written informed consent. The study was approved by the Royal Adelaide Hospital Research Ethics Committee.

The study took place at the Pain and Anaesthesia Research Clinic within the Royal Adelaide Hospital. This study was carried out in accordance with Principles of International Conference on Harmonization Good Clinical Practice, as adopted in Australia, which build upon the ethical codes contained in the Declaration of Helsinki and The Australian National Statement on Ethical Conduct in Research Involving Humans.

An initial test session was performed during screening to familiarize subjects with the four pain assessments. To minimize withdrawals from the study and to ensure recruitment of test drug 'responders', the screening also contained a familiarization event with the injection at the highest dose $(100 \mu \mathrm{g})$ of the HP- $\beta-C D$ formulation into the dominant forearm [4]. At screening an alcohol swab response test was performed to confirm that subjects did not respond with a localized flare due to swabbing of the skin with alcohol.

Each potential participant had to meet the inclusion and exclusion criteria in order to qualify for admission into the study. Key exclusion criteria included pregnancy, breastfeeding, dark skin colour, scarring or tattoos on the arms, regular use of analgesics or subjects suffering from a clinically significant painful condition.

During the trial phase, the eight injections were administered over two occasions. On each occasion the subjects received a total of four injections to the upper and lower forearm sites of both arms. Each injection was separated by $1 \mathrm{~h}$ and $5 \mathrm{~min}$. The two dosing occasions were separated by a minimum of 4 days and each of the 16 subjects was scheduled to receive all eight injections.

The injections were administered according to a randomized Latin Square Design, to ensure that injection site, formulation and dose were balanced.

On each trial day, each subject came in on either mornings or afternoons to decrease the influence of time of day variability within a subject [4]. Subjects rested on a bed and 
superficial skin temperature was fixed at $34-36^{\circ} \mathrm{C}$, using a 250-W infrared heat lamp (Philips, Eindhoven, the Netherlands) positioned about $50 \mathrm{~cm}$ from the subject's volar arm. The temperature was monitored by a thermocouple placed on the skin of the subject, as a fixed temperature has been shown to decrease the variability of the model [7]. Subjects were blindfolded during the assessments.

\section{Capsaicin injections}

Spontaneous pain, flare, allodynia and hyperalgesia were induced by intradermal injection of capsaicin (8-methyl $\mathrm{N}$-vanillyl 6-nonamid) in two different formulations, both administered in strengths $100,30,10$ and $1 \mu \mathrm{g}$. These doses were selected as they are known to be tolerable to subjects while producing areas of allodynia, hyperalgesia, spontaneous pain and flare of sufficient size to be measured accurately [1].

The Tween formulation was prepared according to Simone et al. [3] using a vehicle of Tween $80,7.5 \% \mathrm{w} / \mathrm{v}$ in normal saline. Capsaicin was sourced from Fluka, Switzerland, Batch 21741. The formulation appeared as a solution for the three lowest doses and a colloidal suspension at $100 \mu \mathrm{g}$. The lowest dose, $1 \mu \mathrm{g}$, was considered to be active placebo, since $1 \mu \mathrm{g}$ is reported to produce minimal pain [1] and hence no injections with only the vehicle were given.

The HP- $\beta-C D$ formulation was prepared according to Scanlon et al. [1] with the modification of the concentration of the HP- $\beta-C D$ vehicle, which was $38 \%$ in this study, a concentration known to be equivalent to isotonic solution. The HP- $\beta-C D$ formulation was passed through a sterile syringe filter (Sterivex $0.22 \mu \mathrm{m}$ ) into a sterile vial before syringes were prepared.

In each experiment, a volume of $10 \mu \mathrm{l}$ was injected intradermally into the skin to the midline of either the dominant or nondominant arm and to either forearm or upper arm, avoiding any veins, using a $0.3-\mathrm{ml}$ sterile insulin syringe (BD Ultra-Fine II).

\section{Pharmaceutical analyses}

After the last occasion in the trial, the concentrations of capsaicin in all administered formulations and doses were assayed by high-performance liquid chromatography (HPLC). The neat formulations were diluted 1:1 with mobile phase before $10 \mu \mathrm{l}$ was injected. The isocratic mobile phase was circulated at a flow rate of $1.0 \mathrm{ml} \mathrm{min}^{-1}$ at an ambient temperature of $21^{\circ} \mathrm{C}$. The mobile phase was 75 parts methanol, 25 parts water and 0.1 parts acetic acid and was degassed (500-T degasser; Soniclean, Thebarton, Australia) $30 \mathrm{~min}$ before use. The ultraviolet detector was set to $280 \mathrm{~nm}$. Under these circumstances, capsaicin appeared as a single peak after approximately $8 \mathrm{~min}$.

Stability, adsorption and phase solubility studies were performed on the HP- $\beta-C D$ formulation for development purposes.

\section{Pain assessments}

Information about the assessments and pre-injection assessments were made before the first injection at every occasion and any procedure that might have caused irritation of the skin was avoided before the experiment. The assessments were measured at 5-min intervals for the first $30 \mathrm{~min}$ post injection and then every $10 \mathrm{~min}$ to $1 \mathrm{~h}$ post injection. The assessments of pain were performed in the following order: spontaneous pain, area of flare, allodynia and finally the most invasive measurement, hyperalgesia. One observer performed all measurements of each pain assessment to minimize observer bias.

Spontaneous pain Spontaneous pain was assessed using a $100-\mathrm{mm}$ visual analogue scale (VAS). The scale was calibrated from 0 to 100 , where 0 indicates 'no pain' and 100 indicates the 'worst pain imaginable.' The scores were converted to a numeric scale of $0-100 \mathrm{~mm}$ to correspond to the VAS calibration.

Flare The area of flare was measured by tracing the visually reddened area directly onto a clear acetate. The area was calculated by a digital planimeter (KP-90 N; PLACOM) in $\mathrm{cm}^{2}$.

Allodynia Allodynia was measured with a foam brush (Foam brush 2*; ROYMAC, Newtown, Geelong, Victoria, Australia). The foam brush was stroked in eight compass directions with a speed of $1 \mathrm{~cm} \mathrm{~s}^{-1}$, starting peripherally, about $10 \mathrm{~cm}$ from injection site, and moving inward to the injection site. The volunteer, who had closed eyes, stated when it changed to a more painful condition. The stated points were marked with a water-soluble soft-tipped pen on the skin and then traced onto clear acetate. After the transferring was complete, the dots were washed away with ethanol. All the radii, between injection site and stated dot, were measured with a ruler. Since not all assessments resulted in eight points, an average radius $(\mathrm{mm})$ was used in preference to a calculated area of allodynia and hyperalgesia.

Hyperalgesia The average radius of pin prick-induced hyperalgesia was assessed by applying a standard von Frey hair, number $5.46[1,4]$ with microfilament bending threshold $26 \mathrm{~g}$ (TouchTest 800-821-9319; Semmes Weinstein, Stoelting, IL, USA). The subjects were told to report when the hair caused a greater or changed pain sensation compared with the pinprick sensation felt in the area of normal sensitivity.

The hair was applied in eight compass point directions and assessments started in the area of normal sensitivity, $10 \mathrm{~cm}$ above the injection site. The hair was reapplied at approximately $1-\mathrm{cm}$ intervals every second, moving towards the injection site [3]. The procedure progressed to the site of injection if the subject did not report any 
change in pain state/sensation. The resulting points, demarcating transition from normal sensitivity to hyperalgesia, were traced onto acetate. The transition was made directly after the measurement to decrease the bias. Calculations of the average radius of hyperalgesia were performed as described for allodynia.

\section{Statistical analysis}

All calculations were performed using Microsoft Office Excel Professional edition 2003 (Redmond, WA, USA) and SAS version 9.1 (Cary, NC, USA).

The outcome variables were VAS $(\mathrm{mm})$, flare $\left(\mathrm{cm}^{2}\right)$, allodynia (average radius, $\mathrm{mm}$ ) and hyperalgesia (average radius, $\mathrm{mm}$ ). A mixed model was fitted to each outcome with subject as a random effect and sex (male or female), arm dominance (dominant or nondominant), arm position (upper or lower), formulation (HP- $\beta-C D$ or conventional), dose $(1,10,30$ or $100 \mu \mathrm{g})$ and the interaction of the formulation and the dose each as fixed effects. Nonsignificant terms were dropped from the model.

A $P$-value $<0.05$ was required for statistical significance. Where dose and the interaction between dose and formulation were significant, post hoc tests were performed to determine where the differences occurred and $P$-values were adjusted using the Sidak method to account for multiple testing. Model assumptions were assessed, including normality, and a log transformation of VAS and allodynia was required. For these outcomes means are reported on the log scale and back transformed medians are also reported.

\section{Results}

\section{Population}

Sixteen healthy volunteers entered the trial. Two female subjects withdrew after the first occasion, for reasons not related to adverse events from the capsaicin injections. Their completed data were included in the analysis. Other than pain reported from the injection and measurements, no other adverse events occurred.

\section{Pharmacodynamic data}

Figure 1A-D shows the dose-response data for the four variables. There is a clear dose-response relationship for the HP- $\beta-C D$ formulation for all variables. The values for the Tween formulation were generally lower and with flatter dose-response curves over the range 10-100 $\mu \mathrm{g}$ and especially between 30 and $100 \mu \mathrm{g}$. At $100 \mu \mathrm{g}$ the differences between the two formulations were significant for all variables. At $100 \mu \mathrm{g}$, mean area for hyperalgesia was $9 \mathrm{~cm}^{2}$ [95\% confidence interval $(\mathrm{Cl}) 5,13]$ higher with the HP- $\beta$-CD formulation. Flare area was $5 \mathrm{~cm}^{2}(95 \% \mathrm{Cl} 8,13)$ greater with the HP- $\beta-C D$ formulation.
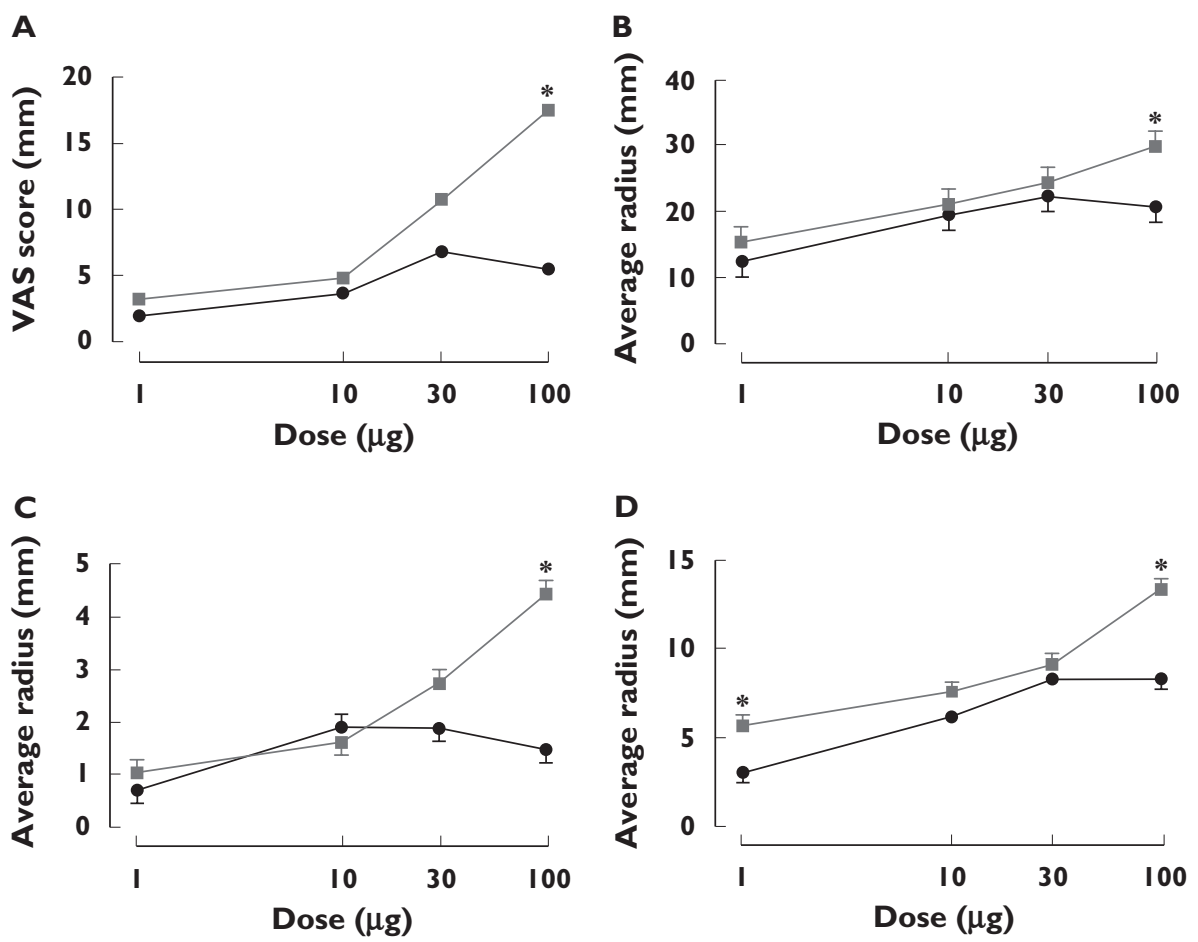

\section{Figure 1}

Dose-response relationships for (A) spontaneous pain by visual analogue scale (VAS), (B) average radius of hyperalgesia, (C) average radius of allodynia and (D) flare area. ${ }^{*} P<0.0001$. Tween (- -$) ; \beta-C D(-)$ 

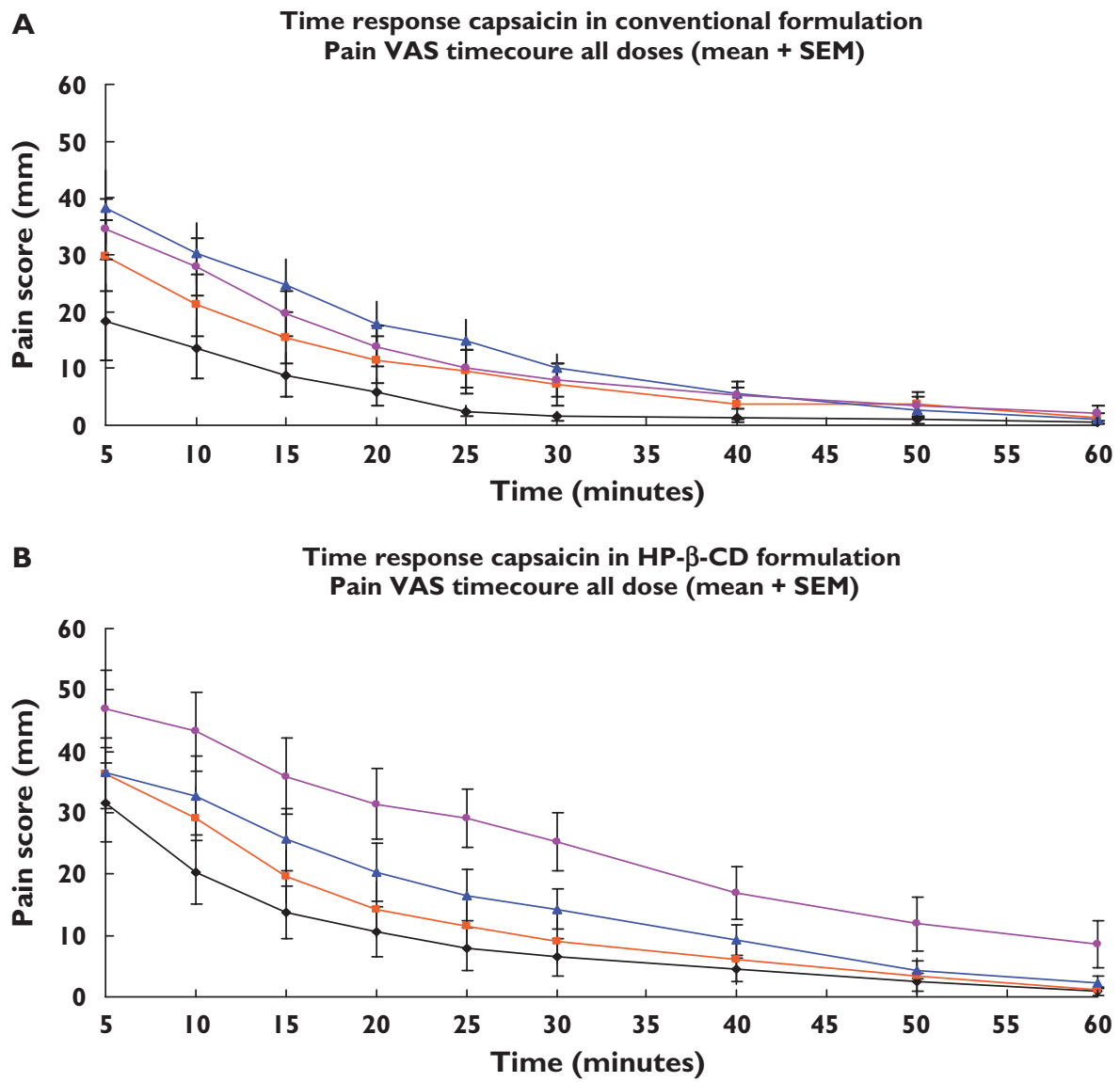

\section{Figure 2}

Time course of spontaneous pain, (A) Tween, conventional formulation. (B) Hydroxypropyl- $\beta$-cyclodextrin (HP- $\beta$-CD) formulation. (A) The mean score of spontaneous pain [rated on visual analogue scale (VAS)] as a function of time. The data are based on all injections of the conventional formulation containing capsaicin dissolved in Tween 80. (B) The mean score of spontaneous pain (rated on VAS) as a function of time. The data are based on all injections of the new formulation containing capsaicin dissolved in HP- $\beta$-CD. $1 \mu \mathrm{g}(--) ; 10 \mu \mathrm{g}(--) ; 30 \mu \mathrm{g}(-\mathbf{\Delta}-) ; 100 \mu \mathrm{g}(-\bullet)$

\section{Table 1}

The overall significance of sex, arm dominance, position, formulation, dose and the interaction of formulation and dose for the four pain assessments (spontaneous pain, flare, allodynia and hyperalgesia)

\begin{tabular}{|c|c|c|c|c|c|c|c|c|}
\hline \multirow[b]{2}{*}{ Effect } & \multicolumn{2}{|c|}{ Spontaneous pain } & \multicolumn{2}{|l|}{ Flare } & \multicolumn{2}{|c|}{ Allodynia } & \multicolumn{2}{|c|}{ Hyperalgesia } \\
\hline & F-value & ProbF & F-value & ProbF & F-value & ProbF & F-value & ProbF \\
\hline Sex & 0.01 & 0.9262 & 0.06 & 0.8013 & 0.91 & 0.3412 & 4.34 & $0.0375^{*}$ \\
\hline Position & 19.39 & $<0.0001$ * & 0.12 & 0.7253 & 30.75 & $<0.0001 *$ & 11.11 & $0.0009^{*}$ \\
\hline Formulation & 51.54 & $<0.0001 *$ & 75.76 & $<0.0001$ * & 31.84 & $<0.0001 *$ & 38.14 & $<0.0001 *$ \\
\hline Dose & 52.99 & $<0.0001$ * & 89.69 & $<0.0001$ * & 34.97 & $<0.0001$ * & 59.13 & $<0.0001^{*}$ \\
\hline Formulation dose & 5.76 & $0.0007 *$ & 10.56 & $<0.0001 *$ & 13.73 & $<0.0001^{*}$ & 7.22 & $<0.0001^{*}$ \\
\hline
\end{tabular}

$* P$-value $<0.05$.

For dose-duration, the same pattern was seen for all four assessments, with the HP- $\beta$-CD formulation producing results of higher magnitude, longer duration and more obvious dose dependence compared with the conventional formulation (VAS data presented in Figure 2A,B).

Table 1 lists the significant covariates for all four outcome variables. Formulation and dose were significant for all variables; dominance for all variables except spontaneous pain; position for all variables except flare; and sex only for hyperalgesia.

\section{HPLC assay}

The results from the HPLC analysis (Table 2) show the accuracy was good for all of the injections except for the $100 \mu \mathrm{g}$ 


\section{Table 2}

The assayed concentration of capsaicin for administered doses $(1,10,30$ and $100 \mu \mathrm{g}$ )

\begin{tabular}{|c|c|c|}
\hline $\begin{array}{l}\text { Labelled concentration } \\
\text { ( } \mu \mathrm{g} \text { per } 10 \mu \mathrm{l})\end{array}$ & $\begin{array}{l}\text { Assayed conce } \\
\text { Conventional } \\
\text { formulation } \\
\text { (accuracy) }\end{array}$ & $\begin{array}{l}\mu g \text { per } 10 \mu \mathrm{l}) \\
\text { HP- } \beta-C D \\
\text { formulation } \\
\text { (accuracy) }\end{array}$ \\
\hline 1 & $1.01(101 \%)$ & $1.13(113 \%)$ \\
\hline 10 & $10.14(101 \%)$ & $12.05(121 \%)$ \\
\hline 30 & $31.04(103 \%)$ & $28.77(96 \%)$ \\
\hline 100 & $38.53(39 \%)$ & 111.12 (111\%) \\
\hline
\end{tabular}

HP- $\beta-C D$, hydroxypropyl- $\beta$-cyclodextrin.

conventional dose, which was much lower than target. The accuracy was better for the conventional formulation in the three lower doses than for the HP- $\beta-C D$ formulation.

\section{Discussion}

In this study, the two formulations produced pharmacodynamically comparable responses for the three lowest doses. The top dose was different between the two formulations and doses $\leq 30 \mu \mathrm{g}$, except that $1 \mu \mathrm{g}$ for flare produced a smaller difference considered to be clinically irrelevant.

The HP- $\beta-C D$ formulation appears to be more suitable for clinical trials since the same dose levels as for the conventional formulation resulted in comparable results for the three lowest doses but longer lasting responses in all four assessments. This is advantageous in clinical trials, since it is desirable to have stable responses with sufficient duration to allow pharmacological intervention without having to administer intolerable amounts of capsaicin. According to the literature, doses of capsaicin up to $250 \mu \mathrm{g}$ have previously been given [4]. The results in this study suggest that it is not necessary to give such large doses since 10,30 and $100 \mu \mathrm{g}$ of the HP- $\beta-C D$ formulation produce satisfactory responses. Using 10 or $30 \mu \mathrm{g}$ may be the most suitable dose levels, since the stimulus from the $100-\mu \mathrm{g}$ dose has been reported to be too intense to detect certain analgesic drug effects [1].

No adverse events other than those anticipated were reported during the trial, suggesting the HP- $\beta$-CD formulation to be as safe and tolerable as the more extensively used Tween formulation. Introduction of new excipients raises potential safety concerns, and nowadays the $\mathrm{HP}-\beta-C D$ vehicle is accepted as safe. In an intravenous human toxicity dosing study, single doses of $3 \mathrm{~g}$ were welltolerated by all volunteers [8] and substitution with hydroxypropyl group is considered to be even safer due to its increased water solubility [9]. The maximum dose of CD in this study was $3.8 \mathrm{mg}$, which is 800 times lower than the maximum well-tolerated dose.
The HPLC assay confirmed the suspicion that the $100-\mu \mathrm{g}$ conventional formulation was a colloidal suspension and crystals were visible at visual inspection. In previous studies with the conventional formulation, an assay has not been performed $[1,3,4]$, or has been performed but without the concentrations clearly stated [5]. Thus, it is unclear whether the maximum solubility of capsaicin in Tween 80 is $39 \mu \mathrm{g} / 10 \mu \mathrm{l}$ or if production of the formulation was unsuccessful at this higher dose level. The low content of soluble capsaicin in the $100-\mu \mathrm{g}$ dose of the Tween formulation is the probable explanation for the reduced response.

Simone et al. [3] reported dose dependence between the 10 - and $100-\mu \mathrm{g}$ doses of the conventional formulation but in their study did not include the critical $30-\mu \mathrm{g}$ dose, a dose supposed to be close to the maximum solubility. Hence, it is not surprising that their study showed dose dependence since they did not cover the range of concentrations where the plateau is reached. Future studies with the conventional formulation have no benefit of injecting higher doses than $39 \mu \mathrm{g}$, since the solution is saturated.

The HP- $\beta-C D$ formulation exhibits clearly advantageous pharmaceutical and production factors in addition to the pattern of more obvious dose dependence for the whole investigated dose range. It is isotonic, easier to produce and gives a better response for allodynia measurements. The most clearly demonstrated advantage for the HP- $\beta-C D$ formulation relates to its ability to remain as a solution at all doses used throughout this study. Dose accuracy is thus optimal when compared with the conventional formulation at the highest dose level. This also enables sterilization through a filter for the HP- $\beta-C D$ formulation. There are also preferable storage conditions for the new formulation. It can be stored in light and at room temperature, which is good compared with the conventional formulation, which needs to be stored in the dark and refrigerated [5]. The HP- $\beta$-CD formulation can thus improve the intradermal capsaicin model, since it has many advantages over the conventional formulation.

The HP- $\beta$-CD formulation produced higher responses in all assessments except one, compared with the conventional formulation. There is no obvious explanation for this finding. Since both formulations were isotonic, the tonicity is probably not the explanation. A possible explanation is the $\mathrm{pH}$ of the formulation. Capsaicin is an agonist at the TRPV1 [10] and this receptor is reported to be activated by $\mathrm{pH} 5.9$ [11]. The reported $\mathrm{pH}$ of the HP- $\beta-C D$ formulation is 6.0 . Hence, this is probably activating the TRPV 1 receptor. A simple $\mathrm{pH}$ assay should be performed in future studies and a study of the HP- $\beta-C D$ vehicle alone should also be implemented to determine the baseline level of the HP- $\beta-C D$ vehicle for all four assessments. A clearly stated $\mathrm{pH}$ is also important for correct comparisons between future results within this model.

Due to the measures taken to reduce environmental causes of variability, including observer bias, within- 
subject variability was low. However, there was significant between-subject variability observed. This demonstrated the importance of cross-over study design instead of a parallel study design. Our study showed that sex had a minor effect and that the arm dominance and arm position influenced the results more than sex. We further confirm the proposal from Hughes et al., where the nondominant arm is reported to be significantly more sensitive to allodynia and hyperalgesia compared with the dominant arm [4].The location of the injection site or the arm had a major effect; the lower arm produced a higher response measuring allodynia, spontaneous pain and hyperalgesia. To our knowledge, no previous studies have investigated the effect of arm injection site. In this study, the approach with arm site was both to investigate the extent of influence of arm site as well as to avoid any reported carryover or desensitizing effects [4]. This study has confirmed that both site and arm dominance must be taken into consideration in study design.

Our study has demonstrated the reported importance of pre-screening of subjects with a test dose of capsaicin. This minimizes withdrawals and ensures adequate responders. The injections given at the screening event resulted in exclusion of three abnormal responders, an observation in accordance with Liu et al. [5].

It is notable and interesting that there are differences in results for flare compared with the three other pain assessments. The probable reason is that flare has another mechanism compared with the other three assessments which also is stated by previous research [12].

Different injection techniques, e.g. angles and depth of the needle, can increase bias and variability. During the trial, a phenomenon with the appearance of a small bleb was seen after some injections. This was a small bubble resulting from the injection volume just under the skin with a confounding appearance similar to flare, and the skin within this small bleb is reported to be hypoalgesic to stimulation from von Frey hair [13].

This study is also consistent with previous studies that have found that allodynia was more short-lived, showed greater variability than hyperalgesia [1,5] and was associated with the presence of poor allodynia responders [5]. A better way of measuring allodynia might need to be investigated, since all factors for decreasing the variability were followed.

This study has provided further information on the performance characteristics of the intradermal capsaicin model and relevant covariates of response. At doses of $\leq 30 \mu \mathrm{g}$, the choice between the Tween-based and HP- $\beta$-CD formulation is unlikely to be important. At higher doses, the HP- $\beta-C D$ formulation is recommended.

\section{Competing interests}

None declared.

This study was funded by the Pain \& Anaesthesia Research Clinic (PARC) of the University of Adelaide.

\section{REFERENCES}

1 Scanlon GC, Wallace MS, Ispirescu JS, Schulteis G. Intradermal capsaicin causes dose-dependent pain, allodynia and hyperalgesia in humans. J Investig Med 2006; 54: 238-44.

2 Pasero C. Pathophysiology of neuropathic pain. Pain Manag Nurs 2004; 5 (4 Suppl. 1): 3-8.

3 Simone DA, Baumann TK, LaMotte RH. Dose-dependent pain and mechanical hyperalgesia in humans after intradermal injection of capsaicin. Pain 1989; 38: 99-107.

4 Hughes A, Macleod A, Growcott J, Thomas I. Assessment of the reproducibility of intradermal administration of capsaicin as a model for inducing human pain. Pain 2002; 99: 323-31.

5 Liu M, Max MB, Robinovitz E, Gracely RH, Bennett GJ. The human capsaicin model of allodynia and hyperalgesia: sources of variability and methods for reduction. J Pain Symptom Manage 1998; 16: 10-20.

6 Challa R, Ahuja A, Ali J, Khar RK. Cyclodextrins in drug delivery: an updated review. AAPS PharmSciTech [electronic resource] 2005; 6: E329-57.

7 Loftsson T, Brewster ME. Pharmaceutical applications of cyclodextrins, 1. Drug solubilization and stabilization. J Pharm Sci 1996; 85: 1017-25.

8 Gould S, Scott RC. 2-Hydroxypropyl- $\beta$-cyclodextrin (HP- $\beta-C D)$ : a toxicology review. Food Chem Toxicol 2005; 43: 1451-9.

9 Stella JV, Rajewski RA. Cyclodextrins. Their future in drug formulation and delivery. Pharm Res 1997; 14: 556-67.

10 Baron R. Mechanisms of disease: neuropathic pain - a clinical perspective. Nat Clin Pract Neurol 2006; 2: 95-104.

11 Levine JD, Alessandri-Haber N. TRP channels: targets for the relief of pain. Biochim Biophys Acta 2007; 1772: 989-1003.

12 Geber C, Fondel R, Krämer HH, Rolke R, Treede RD, Sommer C, Birklein F. Psychophysics, flare, and neurosecretory function in human pain. Models: capsaicin versus electrically evoked pain. J Pain 2007; 8: 503-14.

13 LaMotte RH, Lundberg LE, Torebjörk HE. Pain, hyperalgesia and activity in nociceptive $C$ units in humans after intradermal injection of capsaicin. J Physiol 1992; 448: 749-64. 